\title{
What Is the Plural of a 'Yellow' Anecdote?
}

\author{
Stephen J. W. Evans ${ }^{1}$
}

Published online: 23 November 2015

(c) Springer International Publishing Switzerland 2015

Spontaneous reports (SRs) of suspected adverse drug reactions (ADRs), known as 'Yellow Cards' in the UK for over 50 years, are used in most countries of the world. The total number of countries that have contributed to the World Health Organization (WHO) database of such reports, administered so effectively at the Uppsala Monitoring Centre (UMC), is 122. In 2014 and the first 9 months of 2015, 110 different countries contributed reports, and now there are over 11 million of them. The medical/scientific communities have expressed different views about their value. I recall Tom MacDonald of Dundee saying "The plural of anecdote is not data" in the context of SRs. Others have held views that are the absolute contrary, suggesting they are the strongest evidence when it comes to assessment of harm.

It has been well argued that individual reports can sometimes provide convincing evidence of drug-caused harm. The idea of an anecdote as evidence has been shown by Aronson [1], among others. When it comes to dealing with large numbers of such anecdotes, they have for over 30 years been collected into databases such as that at the UMC. The last two decades have seen development of statistical methods to filter the large number of reports that arrive at regulatory authorities or companies and are included in the databases.

There is probably agreement in the wider community that the occurrence of a number of reports can be 'signals'

Stephen J. W. Evans

stephen.evans@1shtm.ac.uk

1 Department of Medical Statistics, The London School of Hygiene and Tropical Medicine, Keppel Street, London WC1E 7HT, UK of possible harm, though not necessarily sufficient evidence to demonstrate that the adverse event (AE) is a true adverse reaction (caused by the drug).

A good question is whether they are ever anything more than a possible indication of an adverse reaction. The accompanying paper by Maciá-Martínez et al. [2] takes an interesting approach to examine the utility of the statistical methods used to assess them. The authors used the proportional reporting ratio (PRR) [3], which is a simple measure of disproportionality - that is, how much more frequently this combination of a particular drug reported in association with a particular event occurred in the database than might be expected if there were no association between the drug and the event. They calculated this measure for a set of drug/AE combinations that had been subject to regulatory action, so experts had concluded there was reason to believe that the association was causal. In addition, for a drug/AE combination to be included in the series of 15 cases, there had to be accompanying epidemiological evidence, as well as SRs of the association. The authors looked at the relation between the measure of disproportionality and a measure of the relative risk (RR) for that drug from the totality of the available epidemiological evidence. Depending on your prior view, the fairly strong relationship they found may be a surprise or just as expected. It may be noted that, on average, the PRR overestimated the RR.

Starting with cases in which action was taken does not address the issue of whether the primary observation that a PRR (or the value of another measure) is high is evidence of causality on its own. A key phrase used by the authors was "should the signal be confirmed". The magnitude of the PRR may be useful at a stage when further evidence has been gathered to confirm or refute the signal. This study 
could not give the positive predictive value of a raised PRR at any particular threshold, so enthusiasts for SRs must not overinterpret the results. At the same time, those who dismiss SRs as mere anecdotes may need to modify their view.

An important feature of this study was that the reports were counted only up to "the date of the first public awareness of any formal study investigating the same AEdrug were included". The influence of publicity on reporting can severely distort a database and may result in many reports of associations that do not even exist in the population (the purported association between the measles-mumps-rubella vaccine and autism is a wellknown example), but for those where the suspicion of causality may be correct, exaggeration of the effect is likely (e.g. the reported association between cerivastatin and rhabdomyolysis). For associations heavily affected by media attention, it is important to restrict the data to those reported prior to media attention if it occurs earlier than a formal study.

It would appear that there have been few, if any, formal examinations of whether statistical analysis of SRs relates to findings in more rigorous studies. While this relationship between SRs and formal epidemiological results cannot be generalized to all such analyses of SRs, it does suggest that SRs can provide more evidence than cynics have suggested. It is important to note that this uses a disproportionality statistic, not the total number of reports, so it is not evidence that all SRs are to be regarded as signals. In fact, the very use of a disproportionality statistic implies that not all reports are causal. As the authors noted, the total numbers of reports, including non-causal ones, can be seen as a surrogate for the measurement of exposure. There is a choice of cut-off for this statistic so that a "signal of disproportionate reporting" (SDR) [4] is obtained.

It could be argued that the PRR in this instance attempts to estimate the RR of a particular AE with a particular drug in comparison with all other drug users, not similar people who are non-users of the drug. All those not using the drug would, in a population-based study, include people using no drugs at all, as well as possibly some people using other drugs from other classes. As a general rule, we find that RRs in comparison with non-use are higher than those in comparison with use of an alternative drug. However it is generally also acknowledged that the biases in comparison with non-use can be rather greater, so the PRR may be better than one might think. However, this study began with a series of signals that were tested in an observational study. There will have been quite a number of PRRs that were elevated-some substantially so-but for which no observational study was done, perhaps because assessment of the signal suggested that it was unlikely to be causal.
A previous paper [5] showed that SDRs typically have a positive predictive value (referred to as 'precision' in that paper) of less than $30 \%$, and that the different statistical methods can be tuned to give similar results by the choice of the cut-off point to define an SDR. The PRR is simple, but this does not mean that it should be used generally in signal detection, nor will it uniquely be associated with the $\mathrm{RR}$ estimated in more rigorous studies.

One of the first evaluations of the PRR in real data [2] found that medical assessment concluded that $43 \%$ of the SDRs (which were not previously known ADRs) were regarded as being due to the medical condition associated with the indication rather than being due to the drug, so the assessment of SDRs requires more than statistical considerations.

Formally, the reporting odds ratio (ROR) is said to be better than the PRR [6]; the ROR is a measure of the RR under certain conditions, which the PRR technically is not. However, in practice, the PRR and ROR give similar results [7], and in the evaluation by Candore et al. [5], they had virtually identical properties. The PRR is slightly more easily understood by non-statisticians and can be calculated in cases where no estimate of the ROR is obtainable, but where this is so, the data are so sparse than useful conclusions based on the PRR are not easy to obtain.

The reliability of 'lower' levels of evidence may be greater for harm than for benefit. Golder et al. [8] showed that systematic reviews of randomized and observational data give more similar answers than expected.

\section{What Can We Learn from This?}

It is certainly a further justification for statistical analysis of SRs. It does not suggest that 'proper' epidemiological studies are then unnecessary, but as part of a triage process, the magnitude of the PRR may be useful. Waller et al. [9] developed a semi-formal 'impact' analysis with two main factors: a strength of evidence score and a public health consequence score. The PRR (or, rather, one standard error below the PRR itself-and it is not clear if that would have been a better measure here) was a contributor to the strength of the evidence. Seabroke et al. [10] developed this further, using a more complex system of prioritization, and chose a cut-off of 10 for an alternative to the PRRthe empirical Bayes geometric mean [11]—but a cut-off of 3 for the RR from formal studies.

For the 15 signals in the paper by Maciá-Martínez et al. [2], the total number of reports for the drug of concern was reasonably large - the minimum number in EudraVigilance was 679 for cabergoline [M. A. Maciá-Martínez (2015); personal communication]. The number of reports for the combination of an $\mathrm{AE}$ of interest with that drug was nine 
(see case 5 in Table 3 in the paper by Maciá-Martínez et al. [2]). The variance of the PRR or other measures is high when the number of reports for the combination of interest is small, and it can be very unstable when the total for the drug is less than about 100, or if the total number of reports for the $\mathrm{AE}$ is below about 100 . It can be seen that the results from the Spanish Pharmacovigilance Database (FEDRA) show a similar trend but with very much more uncertainty. Some drug/event combinations had zero counts in FEDRA, and for four of the cases with non-zero counts, the total for the drug was less than 100 [M. A. Maciá-Martínez (2015); personal communication]. The most extreme PRRs were cases 5, 6 and 8 in FEDRA, illustrating that the variance of PRR with small numbers is very high. This suggests that one of the 'shrinkage' or Bayesian methods may be more useful; the adaptation of the 'information component' (IC) from the Uppsala group is simple and, combined with the addition of other information in their 'vigiRank' system, may have considerable utility [12]. Whether this will help when the total number of reports is smaller than, say, 100 is not yet known. What is clear is that the extreme PRRs will be 'shrunk' towards unity-for example, case 5 (cabergoline with cardiac valve disease has a PRR of 1290 in FEDRA, but applying the IC leads to a value of about 9).

It turns out that the best evidence for the origin of the phrase about anecdotes not being data is the exact opposite. The originator suggested that a single anecdote was not evidence, but when it is replicated, those anecdotes become data [13]. Maybe there needs to be a paradigm shift to acknowledge that (as was suggested in the New England Journal of Medicine recently in a different context [14]), when handled with care, "Perhaps the plural of anecdote could be data after all".

\section{Compliance with Ethical Standards}

Funding No sources of funding were used in the preparation of this editorial.

Conflict of interest Stephen J. W. Evans has no conflicts of interest that are directly relevant to the content of this editorial.

\section{References}

1. Aronson JK. Unity from diversity: the evidential use of anecdotal reports of adverse drug reactions and interactions. J Eval Clin Pract. 2005;11(2): 195-208.

2. Maciá-Martínez MA, de Abajo FJ, Roberts G, Slattery J, et al. An empirical approach to explore the relationship between measures of disproportionate reporting and relative risks from analytical studies. Drug Saf. 2015. doi:10.1007/s40264-015-0351-3 [Epub ahead of print].

3. Evans SJW, Waller PC, Davis S. Use of proportional reporting ratios (PRRs) for signal generation from spontaneous adverse drug reaction reports. Pharmacoepidemiol Drug Saf. 2001;10(6):483-6.

4. Hauben M, Reich L. Drug-induced pancreatitis: lessons in data mining. Br J Clin Pharmacol. 2004;58(5):560-2.

5. Candore G, Juhlin K, Manlik K, Thakrar B, Quarcoo N, Seabroke $\mathrm{S}$, et al. Comparison of statistical signal detection methods within and across spontaneous reporting databases. Drug Saf. 2015;38(6):577-87.

6. Rothman KJ, Lanes S, Sacks ST. The reporting odds ratio and its advantages over the proportional reporting ratio. Pharmacoepidemiol Drug Saf. 2004;13(8):519-23.

7. Waller P, van Puijenbroek E, Egberts A, Evans S. The reporting odds ratio versus the proportional reporting ratio: 'deuce'. Pharmacoepidemiol Drug Saf. 2004;13(8):525-6.

8. Golder S, Loke YK, Bland M. Comparison of pooled risk estimates for adverse effects from different observational study designs: methodological overview. PLoS One. 2013;8(8):e71813.

9. Waller P, Heeley E, Moseley J. Impact analysis of signals detected from spontaneous adverse drug reaction reporting data. Drug Saf. 2005;28(10):843-50.

10. Seabroke S, Wise L, Waller P. Development of a novel regulatory pharmacovigilance prioritisation system: an evaluation of its performance at the UK Medicines and Healthcare Products Regulatory Agency. Drug Saf. 2013;36(10):1025-32.

11. Almenoff JS, Pattishall EN, Gibbs TG, Dumouchel W, Evans SJW, Yuen N. Novel statistical tools for monitoring the safety of marketed drugs. Clin Pharmacol Ther. 2007;82(2):157-66.

12. Caster O, Juhlin K, Watson S, Norén GN. Improved statistical signal detection in pharmacovigilance by combining multiple strength-ofevidence aspects in vigiRank. Drug Saf. 2014;37(8):617-28.

13. Wolfinger R. The plural of anecdote is data, after all. Revolut Anal. 2011. http://blog.revolutionanalytics.com/2011/04/theplural-of-anecdote-is-data-after-all.html. Accessed 28 Oct 2015.

14. Hunter DJ, D'Agostino RB Sr. Let's not put all our eggs in one basket. N Engl J Med. 2015;373(8):691-3. 